\title{
AVALIAÇÃO DA FLUÊNCIA VERBAL EM CRIANÇAS COM TRANSTORNO DA FALTA DE ATENÇÃO COM HIPERATIVIDADE: UM ESTUDO COMPARATIVO
}

\author{
Verbal fluency evaluation in attention deficit disorder \\ with hyperactivity children: a comparative study \\ Danielle Câmara Silveira (1), Leila Maria Avila Passos (2), \\ Priscila Carla dos Santos ${ }^{(3)}$, Ana Lúcia de Magalhães Leal Chiappetta ${ }^{(4)}$
}

\begin{abstract}
RESUMO
Objetivo: comparar o desempenho de indivíduos com TDAH e grupo controle, em provas de fluência verbal, na faixa etária de 7 aos 12 anos, sem déficit cognitivo. Métodos: foram avaliadas 22 crianças com TDAH e 34 do grupo controle, estudantes de escolas públicas no Estado do Rio de Janeiro. Aplicou-se uma prova de fluência verbal, subdividida em avaliação da fluência fonológica e semântica, sendo utilizada a letra $F$ na prova fonológica e a categoria Animais na prova semântica. Os testes de fluência verbal avaliam o número de palavras produzidas espontaneamente, de forma oral, no período de um minuto. Foram criados quatro intervalos de tempo de 15 segundos procurando estabelecer relação de palavras ditas em cada intervalo por cada grupo. Os participantes foram divididos por idade para facilitar a análise dos resultados. Resultados: não houve diferença relevante entre os sexos. As médias de palavras nas provas fonológica e semântica foram comparadas. Os resultados da prova fonológica foram inferiores aos da semântica, tanto no grupo com TDAH, quanto no grupo controle. Verificou-se, entretanto que os grupos com TDAH e controle apresentaram valores similares nas provas semântica e fonológica, porém com períodos de latência diferentes. Conclusão: os desempenhos de indivíduos com TDAH em provas de fluência verbal fonológica e semântica foram similares aos do grupo controle, e os resultados superiores ocorreram na prova semântica quando comparados com a prova fonológica em ambos os grupos.
\end{abstract}

DESCRITORES: Linguagem; Aprendizagem Verbal; Transtorno da Falta de Atenção com Hiperatividade; Criança

(1) Fonoaudióloga do Corpo de Saúde da Marinha/ Policlínica Naval Nossa Senhora da Glória, PNNSG, Rio de Janeiro, RJ; Especialista em Linguagem pelo CEFAC - Pós-graduação em Saúde e Educação.

(2) Fonoaudióloga do Consultório de Fonoaudiologia Leila Passos; Especialista em Linguagem pelo CEFAC - PósGraduação em Saúde e Educação.

(3) Fonoaudióloga da Prefeitura Municipal de Itaguaí, Itaguaí, RJ; Especialista em Linguagem pelo CEFAC - PósGraduação em Saúde e Educação.

(4) Fonoaudióloga do Setor de Investigação em Doenças Neuromusculares da Universidade Federal de São Paulo, UNIFESP, São Paulo, SP; Doutora em Ciências pela Universidade Federal de São Paulo.

Conflito de interesses: inexistente

\section{INTRODUÇÃO}

O Transtorno de Déficit de Atenção e Hiperatividade (TDAH) é o transtorno neuropsiquiátrico mais frequentemente diagnosticado na infância, podendo persistir até a idade adulta em torno de 60 a $70 \%$ dos casos. São características do quadro: a desatenção, a agitação psicomotora e a impulsividade que podem variar em maior ou menor grau, de acordo com o subtipo, a saber: predominantemente desatento; predominantemente hiperativo/ impulsivo ou combinado. O TDAH possui uma forte predisposição hereditária podendo ser agravado 
por fatores ambientais. A prevalência varia entre 5 a $15 \%$ das crianças em idade escolar e a incidência é três vezes maior no sexo masculino. Estudos epidemiológicos mais rigorosos definiram taxas de $4 \mathrm{a}$ $12 \%$ da população geral de crianças de 6 a 12 anos de idade ${ }^{1-8}$.

Dentre os pacientes com diagnóstico de TDAH, $44 \%$ apresentam outra comorbidade psiquiátrica. Normalmente, entre 20 e $40 \%$ das pessoas com TDAH apresentam distúrbios de humor, depressão e ansiedade. A dislexia é um dos transtornos específicos do aprendizado. Em crianças com TDAH tais transtornos situam-se entre 20 a $80 \%$. O histórico de atraso de linguagem em crianças com TDAH é de $6 \%$ a $35 \%$, enquanto que na população sem TDAH é de $2 \%$ a $6 \%{ }^{9}$. Pesquisas preliminares em TDAH indicam a presença de alterações em lobos frontais, corpo caloso, gânglios de base e cerebelo. Indivíduos com TDAH tendem a apresentar características bastante semelhantes àquelas decorrentes de lesões na área pré-frontal. As relações entre linguagem e atenção são evidentes. $O$ paciente com TDAH apresenta desvantagem em lidar com o processo de tomada e manutenção de turnos na conversa diária. O TDAH acarreta uma mudança na vida dos indivíduos que vai interferir e repercutir nas atividades cotidianas, na aprendizagem escolar e nas relações sociais ${ }^{1,3-5,8,9}$.

Os pacientes com TDAH apresentam alterações específicas em uma função cognitiva chamada de função executiva. A função executiva abrange todos os processos responsáveis por planejar, focalizar, guiar, direcionar e integrar as funções cognitivas, destacando: o estado de alerta, atenção sustentada e seletiva, tempo de reação, fluência e a flexibilidade do pensamento, além de emoções e comportamentos ${ }^{1,10-12}$. As funções executivas estão entre os aspectos mais complexos da cognição ${ }^{13}$. Déficits relacionados a funções executivas são amplamente descritos nos subtipos de TDAH embora nem sempre os testes neuropsicológicos consigam retratar os comprometimentos que se manifestam no dia-adia dos sujeitos ${ }^{14}$.

A fluência verbal tem sido largamente empregada na avaliação das funções executivas. Os testes de fluência verbal avaliam a maneira como os sujeitos organizam seus pensamentos envolvendo velocidade de produção lexical e acesso lexical automático. As provas de fluência verbal (semântica e fonológica) são usadas para avaliar a capacidade de armazenamento semântico, a habilidade de recuperar as informações e identificação das funções executivas ${ }^{15}$. As respostas dependem do nível de inteligência, vocabulário e atenção. São necessários ainda componentes de memória de trabalho para que o indivíduo não persevere nas respostas. Problemas de fluência geralmente prejudicam a fala, leitura e escrita ${ }^{11,16,17}$. Diversas pesquisas analisaram, através de exames de imagem, a fluência verbal de indivíduos normais e com patologias psiquiátricas e neurológicas, as áreas cerebrais ativadas foram de lobo frontal ${ }^{11,13,15-18}$.

O objetivo desta pesquisa é comparar os desempenhos de indivíduos com TDAH e grupo controle, em provas de fluência verbal, na faixa etária de 7 aos 12 anos, sem déficit cognitivo.

\section{MÉTODOS}

Foi realizado um estudo transversal em alunos de escolas públicas, de ambos os sexos, dos sete aos 12 anos, no Estado do Rio de Janeiro.

As autoras optaram por aplicar uma prova de fluência verbal, subdividida em avaliação da fluência fonológica e semântica. Os testes de fluência verbal avaliam o número de palavras produzidas espontaneamente, de forma oral, em um determinado período de tempo (1 minuto) e dentro de uma categoria limitada, fonológica (palavras começadas com F, A, S) e semântica (palavras que pertençam a classe frutas ou animais) ${ }^{19}$. O teste foi aplicado individualmente, de forma parcial, usando a letra "F" para avaliar fluência fonológica e a categoria "animais" para análise semântica. A letra "F" foi escoIhida por apresentar maior frequência no português do Brasil, junto com as letras "C, P, M e L" ${ }^{20}$. O examinador esclareceu que seriam desconsiderados nomes próprios, números e palavras que divergissem apenas pelo sufixo. A amostra foi composta por 22 indivíduos com TDAH, sendo 5 meninas e 17 meninos e 34 do grupo controle, 20 meninas e 14 meninos.

Foram criados quatro intervalos de tempo de 15 segundos procurando estabelecer relação de palavras ditas em cada intervalo por cada grupo.

As crianças com TDAH testadas foram previamente avaliadas por profissional competente, aplicando-se o "Teste de Matrizes Progressivas de Raven". Este teste avalia o raciocínio não verbal e consiste em pranchas contendo uma figura modelo e alternativas de escolha para que o examinando assinale a alternativa que corresponde ao modelo. Como a tarefa da criança se resume em assinalar a alternativa desejada, o teste permite avaliar a habilidade de raciocínio independentemente de respostas verbais. O objetivo do teste foi padronizar os grupos excluindo prejuízos cognitivos que pudessem prejudicar a amostra ${ }^{21}$.

A amostra analisada foi composta pelas respostas dos participantes selecionados entre todos que atendessem aos seguintes critérios de inclusão: possuir o Termo de Consentimento Livre e Esclare- 
cido assinado por seus responsáveis legais, autorizando a sua participação e concordar em participar da pesquisa; não apresentar quaisquer outros comprometimentos clínicos tais como, déficits auditivos, visuais sem correção, deficiência mental e outras patologias psiquiátricas e/ou neurológicas.

Os pacientes com TDAH foram diagnosticados em avaliação psiquiátrica, segundo critérios do Manual Diagnóstico e Estatístico de Transtornos Mentais, $4^{a}$ edição (DSM-IV) e os responsáveis preencheram um questionário denominado MTA SNAP IV Escala para pais e professores ${ }^{22}$. O questionário também foi respondido pelo grupo controle a fim de evitar crianças com TDAH não diagnosticadas.

Os sujeitos foram divididos em dois grupos, levando-se em consideração a faixa etária: Grupo 1 participantes com idades entre sete e 9,6 anos; Grupo 2 participantes com idades entre 9,7 e 12 anos.

No momento da coleta de dados os participantes com TDAH estavam sem medicação específica por mais de 12 horas.

Os resultados dos indivíduos com TDAH foram comparados com os indivíduos sem TDAH procurando se estabelecer uma relação entre prejuízos de fluência verbal e déficit de atenção.

Este projeto foi aprovado pelo Comitê de Ética em Pesquisa com Seres Humanos do CEFAC Pós-Graduação em Saúde e Educação, sob número 096/07.

Analisaram-se os dados de forma quantitativa e qualitativa. Para o estudo estatístico foram realizados os testes de Mann Whitney. Na presente pesquisa foi considerado um nível de significância de $5 \%$, sendo todos os intervalos de confiança construídos com $95 \%$ de confiança estatística.

\section{RESULTADOS}

Não houve diferença relevante entre os sexos no desempenho das provas considerando o $p$-valor inferior a 0,05. Na prova fonológica a média de palavras de meninos com TDAH foi de 5,71 e meninas de 6,80 com p-valor de 0,3234. O grupo controle atingiu média de palavras 7,07 para meninos e 7,30 de palavras para meninas com p-valor 0,8474 . $\mathrm{Na}$ prova semântica os valores médios para meninos com TDAH foram de 12,29 e meninas $11,40 \mathrm{com}$ p-valor 0,6646 já no grupo controle, a média para meninos foi 12,86 e meninas 12,95 com $p$-valor 1,000 . As médias de palavras nas provas fonológica e semântica foram comparadas. Os resultados da prova fonológica foram inferiores aos da semântica, tanto no grupo com TDAH, quanto no grupo controle (Tabelas 1, 2, 3 e 4).

O grupo com TDAH apresentou resultados próximos aos do grupo controle em ambas as provas com p-valor superior a 0,05.

Os subtipos de TDAH, a saber: predominante desatento $(\mathrm{N}=5,23 \%)$, hiperativo/ impulsivo $(\mathrm{N}=6$, $27 \%$ ) ou combinado ( $N=11,50 \%)$ foram analisados entre si e o grupo controle.

O grupo controle obteve desempenho superior nos primeiros 15 segundos na avaliação fonológica quando comparado ao TDAH do subtipo combinado (Tabela 5). O desempenho do grupo controle foi inferior nos últimos 15 segundos ao ser comparado com o subtipo hiperativo/Impulsivo (Tabela 6).

No que diz respeito à prova semântica, o grupo controle obteve respostas superiores apenas nos primeiros 15 segundos, média de 5,94 em relação ao grupo com TDAH, do subtipo desatento média de 3,33 palavras $p$-valor 0,0019 . Na média final houve recuperação do grupo com TDAH e o $p$-valor final foi de 0,0794.

Tabela 1 - Resultados dos grupos com TDAH e controle na prova fonológica, por faixa etária

\begin{tabular}{clcccccc}
\hline SEPARAÇÃO DOS GRUPOS & \multicolumn{3}{c}{$\mathbf{7}$ a 9,6 meses } & \multicolumn{3}{c}{$\mathbf{9 , 7}$ a 12 anos } \\
\cline { 2 - 7 } POR IDADE & Média & DP & p-valor & Média & DP & p-valor \\
\hline 0 0-15" & TDAH & 2,44 & 1,81 & 0,2272 & 3,08 & 0,95 & 0,1321 \\
& Controle & 3,44 & 1,67 & & 3,78 & 1,44 & \\
$16-30 "$ & TDAH & 1,22 & 0,83 & 1,0000 & 1,31 & 1,25 & 0,2678 \\
& Controle & 1,25 & 1,06 & & 1,94 & 1,55 & \\
$31-45 "$ & TDAH & 1,00 & 1,12 & 1,0000 & 0,77 & 0,83 & 0,2224 \\
& Controle & 0,94 & 0,85 & & 1,22 & 1,00 & \\
$46-60 "$ & TDAH & 1,44 & 1,24 & 0,5436 & 1,15 & 0,80 & 0,1259 \\
& Controle & 1,00 & 0,82 & & 0,78 & 1,00 & \\
\multirow{2}{*}{ Total de palavras } & TDAH & 5,44 & 2,79 & 0,4719 & 6,31 & 2,53 & 0,1323 \\
& Controle & 6,63 & 2,55 & & 7,72 & 2,08 & \\
\hline
\end{tabular}

TDAH N=22; Controle N=34; DP= desvio padrão; $p<0,05$ (significante) testes de Mann Whitney

Rev CEFAC, v.11, Supl2, 208-216, 2009 
Tabela 2 - Resultados dos grupos com TDAH e controle na prova semântica, por faixa etária

\begin{tabular}{|c|c|c|c|c|c|c|c|}
\hline \multirow{2}{*}{\multicolumn{2}{|c|}{$\begin{array}{c}\text { SEPARAÇÃO DOS GRUPOS } \\
\text { POR IDADE }\end{array}$}} & \multicolumn{3}{|c|}{7 a 9,6 meses } & \multicolumn{3}{|c|}{9,7 a 12 anos } \\
\hline & & \multirow{2}{*}{$\begin{array}{l}\text { Média } \\
4,22\end{array}$} & \multirow{2}{*}{$\begin{array}{c}\text { DP } \\
1,86\end{array}$} & \multirow{2}{*}{$\begin{array}{l}\text { p-valor } \\
0,0499\end{array}$} & \multirow{2}{*}{$\begin{array}{l}\text { Média } \\
4,92\end{array}$} & \multirow{2}{*}{$\begin{array}{c}\text { DP } \\
1,66\end{array}$} & \multirow{2}{*}{$\begin{array}{r}\text { p-valor } \\
0,0681\end{array}$} \\
\hline $0-15^{\prime \prime}$ & TDAH & & & & & & \\
\hline & Controle & 5,75 & 1,48 & & 6,11 & 1,32 & \\
\hline \multirow[t]{2}{*}{ 16-30" } & TDAH & 3,67 & 2,65 & 0,8627 & 3,77 & 1,48 & 0,7584 \\
\hline & Controle & 3,19 & 1,28 & & 3,56 & 1,82 & \\
\hline \multirow[t]{2}{*}{$31-45^{\prime \prime}$} & TDAH & 2,00 & 1,00 & 0,9068 & 1,85 & 0,90 & 0,5291 \\
\hline & Controle & 2,06 & 1,34 & & 2,06 & 1,11 & \\
\hline \multirow[t]{2}{*}{$46-60 "$} & TDAH & 1,67 & 1,22 & 0,3342 & 1,92 & 1,04 & 0,6643 \\
\hline & Controle & 1,19 & 1,05 & & 1,89 & 1,71 & \\
\hline \multirow[t]{2}{*}{ Total de palavras } & TDAH & 11,56 & 3,00 & 0,7309 & 12,46 & 2,88 & 0,4437 \\
\hline & Controle & 12,19 & 2,64 & & 13,56 & 3,36 & \\
\hline
\end{tabular}

TDAH N=22; Controle N=34; $\mathrm{DP}=$ desvio padrão; $\mathrm{p}<0,05$ (significante) testes de Mann Whitney

Tabela 3 - Resultados do grupo com TDAH nas provas fonológica e semântica

\begin{tabular}{cccccccc}
\hline \multirow{2}{*}{$\begin{array}{c}\text { SEPARAČ̃O DOS GRUPOS } \\
\text { POR IDADE }\end{array}$} & \multicolumn{3}{c}{ 7 a 9,6 meses } & \multicolumn{3}{c}{$\mathbf{9 , 7}$ a 12 anos } \\
\cline { 2 - 7 } & & Média & DP & p-valor & Média & DP & p-valor \\
\hline $0-15^{\prime \prime}$ & $\mathrm{F}$ & 2,44 & 1,81 & 0,0705 & 3,08 & 0,95 & 0,0052 \\
& $\mathrm{~S}$ & 4,22 & 1,86 & & 4,92 & 1,66 & \\
$16-30 "$ & $\mathrm{~F}$ & 1,22 & 0,83 & 0,0276 & 1,31 & 1,25 & 0,0004 \\
& $\mathrm{~S}$ & 3,67 & 2,65 & & 3,77 & 1,48 & \\
$31-45^{\prime \prime}$ & $\mathrm{F}$ & 1,00 & 1,12 & 0,074 & 0,77 & 0,83 & 0,0075 \\
& $\mathrm{~S}$ & 2,00 & 1,00 & & 1,85 & 0,90 & \\
$46-60 "$ & $\mathrm{~F}$ & 1,44 & 1,24 & 0,675 & 1,15 & 0,80 & 0,0527 \\
& $\mathrm{~S}$ & 1,67 & 1,22 & & 1,92 & 1,04 & \\
Total de palavras & $\mathrm{F}$ & 5,44 & 2,79 & 0,0018 & 6,31 & 2,53 & 0,0002 \\
& $\mathrm{~S}$ & 11,56 & 3,00 & & 12,46 & 2,88 & \\
\hline
\end{tabular}

$\mathrm{F}=$ fonológica; $\mathrm{S}=$ semântica; $\mathrm{p}<0,05$ (significante) testes de Mann Whitney

Tabela 4 - Resultados do grupo Controle nas provas fonológica e semântica

\begin{tabular}{cccccccc}
\hline \multirow{2}{*}{$\begin{array}{c}\text { SEPARAÇÃO DOS GR UPOS } \\
\text { POR IDADE }\end{array}$} & \multicolumn{3}{c}{ 7 a 9,6 meses } & \multicolumn{3}{c}{$\mathbf{9 , 7}$ a 12 anos } \\
\cline { 2 - 7 } & & Média & DP & p-valor & Média & DP & p-valor \\
\hline 0-15" & $\mathrm{F}$ & 3,44 & 1,67 & 0,0009 & 3,78 & 1,44 & 0,0001 \\
& $\mathrm{~S}$ & 5,75 & 1,48 & & 6,11 & 1,32 & \\
$16-30 "$ & $\mathrm{~F}$ & 1,25 & 1,06 & 0,0004 & 1,94 & 1,55 & 0,0129 \\
& $\mathrm{~S}$ & 3,19 & 1,28 & & 3,56 & 1,82 & \\
$31-45^{\prime \prime}$ & $\mathrm{F}$ & 0,94 & 0,85 & 0,0155 & 1,22 & 1,00 & 0,0275 \\
& $\mathrm{~S}$ & 2,06 & 1,34 & & 2,06 & 1,11 & \\
$46-60 "$ & $\mathrm{~F}$ & 1,00 & 0,82 & 0,6923 & 0,78 & 1,00 & 0,0284 \\
& $\mathrm{~S}$ & 1,19 & 1,05 & & 1,89 & 1,71 & \\
Total de palavras & $\mathrm{F}$ & 6,63 & 2,55 & 0 & 7,72 & 2,08 & 0 \\
& $\mathrm{~S}$ & 12,19 & 2,64 & & 13,56 & 3,36 & \\
\hline
\end{tabular}

$\mathrm{F}=$ fonológica; $\mathrm{S}=$ semântica; $\mathrm{p}<0,05$ (significante) testes de Mann Whitney 
Tabela 5 - Desempenho do grupo Controle na prova fonológica comparado ao TDAH subtipo combinado (7 a 12 anos)

\begin{tabular}{|c|c|c|c|c|}
\hline TDAH C $\mathrm{N}=11 /$ Controle $\mathrm{N}=34$ & & Média & Desvio padrão & p-valor \\
\hline \multirow[t]{2}{*}{$0-15 "$} & TDAH C & 2,36 & 1,36 & 0,0206 \\
\hline & Controle & 3,62 & 1,42 & \\
\hline \multirow[t]{2}{*}{ 16-30" } & TDAH C & 1,36 & 1,36 & 0,5957 \\
\hline & Controle & 1,79 & 1,37 & \\
\hline \multirow[t]{2}{*}{$31-45^{\prime \prime}$} & TDAH C & 1,36 & 1,03 & 0,4059 \\
\hline & Controle & 1,31 & 0,97 & \\
\hline \multirow[t]{2}{*}{$46-60 "$} & TDAH C & 1,09 & 1,14 & 0,642 \\
\hline & Controle & 1,07 & 1,03 & \\
\hline \multirow[t]{2}{*}{ Total de palavras } & TDAH C & 5,64 & 3,26 & 0,1388 \\
\hline & Controle & 7,59 & 2,24 & \\
\hline
\end{tabular}

TDAH C= subtipo combinado; $p<0,05$ (significante); testes de Mann Whitney

Tabela 6 - Desempenho do grupo Controle na prova fonológica comparado ao TDAH subtipo hiperativo/impulsivo (7 a 12 anos)

\begin{tabular}{ccccc}
\hline TDAH H N=6/Controle $\mathbf{N = 3 4}$ & & Média & Desvio padrão & p-valor \\
\hline 0-15" & TDAH H & 3,33 & 1,51 & 0,7262 \\
& Controle & 3,62 & 1,42 & \\
$16-30 "$ & TDAH H & 1,17 & 0,75 & 0,5202 \\
& Controle & 1,79 & 1,37 & \\
\multirow{2}{*}{1 -45" } & TDAH H & 0,17 & 0,41 & 0,0212 \\
& Controle & 1,31 & 0,97 & \multirow{2}{*}{0,0359} \\
\multirow{2}{*}{ 46-60" } & TDAH H & 1,83 & 0,98 & \multirow{2}{*}{0,5522} \\
& Controle & 1,07 & 1,03 & \\
\hline
\end{tabular}

TDAH H= subtipo hiperativo/impulsivo; $\mathrm{p}<0,05$ (significante); testes de Mann Whitney

\section{DISCUSSÃO}

Os déficits em funções executivas são amplamente estudados em indivíduos com diagnóstico de TDAH. Os prejuízos são relacionados às áreas cerebrais envolvidas e as questões atencionais ${ }^{13}$.

Este estudo enfocou crianças, de ambos os sexos comparando pacientes com diagnóstico de TDAH de diversos subtipos e um grupo controle, falantes do português. Quando analisados os resultados dos grupos TDAH e controle em ambas as provas não se verificou diferenças significantes, assim como em estudo holandês com crianças com TDAH de faixa etária aproximada ${ }^{23}$. Alguns autores detectaram prejuízos na fluência verbal em sujeitos com TDAH ${ }^{24}$, no entanto há divergências na literatura quanto aos déficits em função executiva ${ }^{11,13,25}$. Nesta pesquisa os resultados de maior relevância foram aqueles que compararam fluência verbal fonológica e semântica entre si em ambos os grupos.

Quando se levam em consideração as áreas cerebrais envolvidas, observa-se que a fluência fonológica está associada à região frontal, menos dependente da memória e mais relacionada com uma efetiva capacidade de iniciação e estratégia de busca, um processo mais laborioso, já a fluência semântica está relacionada à região temporal e requer atributos de memória e armazenamento verbal ${ }^{13,15,17,19,26-30}$, cujo processo é mais automatizado. Crianças com lesões em hemisfério esquerdo em lobos frontal e temporal, além de crianças disléxicas apresentam rebaixamento nas provas de fluência verbal ${ }^{25}$.

As diferenças nos desempenhos em tarefas semânticas e fonológicas sugerem que a linguagem seja representada semanticamente e não alfabeticamente ${ }^{11}$. Há diferenças na prova semântica, quando o objeto solicitado é animado (ex: animais) 
ou inanimado (ex:vestuário) por envolverem sensações, tato e outras experiências emocionais, que estimulam regiões cerebrais diferentes.

Em crianças sem patologias foram encontrados valores de referência de fluência verbal nos idiomas: Hebraico, Inglês, Italiano, Francês e Alemão, na faixa etária até 12 anos, cujos valores foram: 6-8 palavras na prova fonológica e 11-15 palavras na prova semântica ${ }^{31}$.

Quanto ao aspecto idade, neste estudo com crianças em fase de desenvolvimento linguístico verificou-se que a fluência verbal progride conforme a evolução da mesma, fato este identificado em estudo americano ${ }^{25}$ e hebraico ${ }^{31}$, o que significa melhores estratégias de organização. Em estudo espanhol com adultos jovens sem patologias, a idade contribuiu na fluência semântica e o tempo de escolaridade influenciou nas respostas fonológicas mais acentuadamente 15,32-34, contudo em estudo posterior, verificou-se a influência de ambos os aspectos nas provas semânticas ${ }^{35}$. No Brasil, apenas o aspecto escolaridade interferiu nos resultados em prova semântica, categoria Animais ${ }^{19}$. Os pontos de cortes de estudos com adultos no Brasil são: 9 animais/minuto: até 8 anos de escolaridade, e 13 animais/minuto: acima de 8 anos de escolaridade ${ }^{36}$. A comparação de resultados com outros idiomas pode ser prejudicada devido à diferença no tamanho médio das palavras, uma vez que a prova relaciona número de produções em um determinado intervalo de tempo.

Em praticamente toda a literatura pesquisada as médias de palavras na fluência semântica são maiores que na fonológica 15,17,19,31,32,37 inclusive em casos de esquizofrenia ${ }^{26-28}$. Em contrapartida, na doença de Alzheimer, os pacientes apresentaram maior facilidade na prova fonológica. Conforme agravamento da doença, ocorreu prejuízo nas duas provas ${ }^{28}$. Pacientes com Esclerose Lateral Amiotrófica com déficits em funções executivas também obtiveram resultados rebaixados em fluência fonológica ${ }^{29}$.

A despeito de não ter sido encontradas diferenças no número total de palavras ditas por meninos e meninas, estudos de neuroimagem sugerem que homens e mulheres usam diferentes estratégias na produção de palavras ${ }^{31}$.

No grupo com TDAH, houve prevalência no número de meninos, o que pode ser justificado pela baixa demanda de encaminhamentos de meninas para diagnóstico e tratamento especializado ${ }^{7}$.

Nesta pesquisa, as crianças com TDAH foram divididas em subtipos a fim de verificar a influência das diferentes modalidades cognitivas encontradas em cada subtipo. Autores observaram que os tipos combinado e desatento tendem a apresentar déficits neuropsicológicos semelhantes ${ }^{38}$, sendo o subtipo desatento aquele que apresentaria maiores prejuízos em testes neuropsicológicos. Numa amostra de crianças brasileiras com TDAH, os autores sugerem que o desempenho neuropsicológico do subtipo combinado é inferior aos demais apenas quando se empregam tarefas mais longas, exigindo maiores níveis de sustentação ${ }^{38}$. Neste estudo os resultados finais entre os subtipos foram pouco relevantes, porém o fator tempo foi um diferencial encontrado e sugere que o funcionamento do TDAH seja prejudicado nas habilidades de processamento da informação verbal abstrata ${ }^{11}$.

Apesar das médias de palavras ao final das provas serem similares, as crianças com TDAH precisaram de um tempo adicional para iniciar as respostas /desencadear o processo, o que pode justificar os piores resultados nos primeiros 15 segundos das provas. Este achado coincide com outros trabalhos em população de crianças com TDAH ${ }^{11,39}$. Quanto à latência nas respostas, crianças israelenses obtiveram latência diminuída para falar a $1^{\underline{a}}$ palavra na prova semântica ${ }^{31}$. Numa pesquisa brasileira em pacientes com doença de Parkinson observou-se redução da latência no tempo de reação simples e redução na variação do tempo de reação aos estímulos. A reposição de levodopa pode melhorar a atenção e/ou funções executivas, envolvidas nos ganhos cognitivos observados em indivíduos acometidos pelo Parkinson ${ }^{40}$.

Em ambas as provas, o grupo controle apresentou maior número de palavras nos primeiros 15 segundos com posterior decréscimo. Este fato ocorreu em estudos com população adulta, sem patologias nos idiomas espanhol ${ }^{32}$ e português ${ }^{19}$. Estes resultados são confirmados por estudos anteriores que afirmam que a geração de palavras nas tarefas de fluência tende a ocorrer em jatos, e com frequência são semanticamente relacionadas ${ }^{41}$.

A alta distractibilidade dos indivíduos com déficit de atenção é descrita na literatura, no entanto, autores sugerem que de fato eles se desinteressam com maior facilidade ${ }^{39}$. Foi observado que o fator motivacional muito contribuiu com as respostas nas provas, pois as crianças encararam a tarefa como um desafio. Este fator pode ter influenciado o resultado final da avaliação. Os resultados das pesquisas que abordam o tema TDAH e fluência verbal são divergentes e talvez fossem necessárias avaliações com maior número de sujeitos e com períodos de tempo mais amplos a fim de comprovar os déficits em funções executivas descritos na literatura. 


\section{CONCLUSÃO}

Diante do estudo realizado, é possível concluir que os desempenhos de indivíduos com TDAH em provas de fluência verbal fonológica e semântica são similares aos do grupo controle, porém com características diferentes e os melhores resultados ocorrem na prova semântica quando comparados com a prova fonológica em ambos os grupos.

\section{AGRADECIMENTOS}

Agradecemos a Direção da Policlínica Naval N Sra da Glória, seus usuários e a Escola Municipal C.C.Santos Anjos pela autorização para realização da pesquisa, e a psicóloga Elaine S. Martins Caiado pela forte contribuição nas diversas etapas do trabalho.

\section{ABSTRACT}

Purpose: to compare the performances of individuals with ADHD and control group, in verbal fluency tests, from 7 to 12 years, without cognitive deficit. Methods: 22 children with ADHD and 34 control group had been evaluated, students of a public school in the State of Rio de Janeiro. A verbal fluency test was applied, subdivided in evaluation of the phonological fluency, using the letter $F$ and semantics, using Animal category in the semantic test. The verbal fluency tests evaluate the number of verballyproduced words, in just one minute period. Four intervals of 15 seconds had been created looking for setting up the relation of words said in each interval for each group. The participants had been divided by age to make easier the analysis of the results. Results: no difference between genders was found. The average number of words in phonological and semantics tests had been compared. The performance of the phonological test had been lower than semantics in both groups. The ADHD group presented similar results to the control group in both tests, but with different latency periods. Conclusion: the performances of ADHD in phonological verbal fluency and semantics had been similar to the control group, and the higher results had occurred in the semantics tests in both groups.

KEYWORDS: Language; Verbal Learning; Attention Deficit Disorder with Hyperactivity; Child

\section{REFERÊNCIAS}

1. Capovilla AGS, Cozza HFZ, Capovilla FC, Macedo EC. Funções executivas em crianças e correlação com desatenção e hiperatividade. Temas Desenvolv. 2005; 14(82):4-14.

2. Kummer A, Teixeira Júnior AL, Costa C. Possíveis endofenótipos em transtornos de déficit de atenção/ hiperatividade. Rev Psiq Psic Crianças Adolesc. 2006; 12(6):32-5.

3. Mattos $P$, Palmini A, Salgado CA, Segenreich D, Grevet E, Oliveira IR, et al. Painel brasileiro de especialistas sobre diagnóstico do transtorno de déficit de atenção/hiperatividade (TDAH) em adultos. Rev Psiquiatr RS. 2006; 28(1):50-60.

4. Moraes C, Ciasca SM, Ribeiro MVM. Problemas escolares e sua associação com transtorno de déficit de atenção e hiperatividade. In: Valle LELR, organizador. Neuropsiquiatria: infância e adolescência. 1.ed. Poços de Caldas: ABENEPI. Editoração eletrônica: Tera Dorea; 2006. p. 33-40.

5. Pereira HS, Araújo APQC, Mattos P. Transtorno do déficit de atenção e hiperatividade (TDAH): aspectos relacionados à comorbidade com distúrbios da atividade motora. Rev Bras Saúde Matern Infant. 2005; 5(4):391-402.

6. Vasconcelos MM, Werner Júnior J, Malheiros AFA, Lima DFN, Santos ISO, Barbosa JB. Prevalência do transtorno de déficit de atenção/ hiperatividade numa escola pública primária. Arq Neuropsiquiatr. 2003; 61(1):67-73.

7. Vera CFD, Conde GES, Wajnsztejn R, Nemr K. Transtornos de aprendizagem de presença de respiração oral em indivíduos com diagnóstico de transtorno de déficit de atenção/hiperatividade (TDAH). Rev CEFAC. 2006; 8(4):441-55.

8. Pastura GMC, Mattos P, Araújo APQC. Desempenho escolar e transtorno do déficit de atenção e hiperatividade. Rev Psiq Clin. 2005; 32(6):324-9.

9. Lima CC, Albuquerque G. Avaliação de linguagem e co-morbidade com transtornos de linguagem. In: Rohde LA, Mattos P. Princípios e práticas em TDAH. Porto Alegre: Artmed; 2003. p.117-42.

10. Grevet EH, Abreu PB, Shansis F. Proposta de uma abordagem psicoeducacional em grupos 
para pacientes adultos com transtorno de déficit de atenção/hiperatividade. Rev Psiquiatr. 2003; 25(3):446-52.

11. Hurks PPM, Hendriksen JGM, Vles JSH, Kalff AC, Feron FJM, Kroes M, et al. Verbal fluency over time as a measure of automatic and controlled processing in children with ADHD. Brain and Cognition. 2004; 55(3):535-44.

12. Hamdan AC, Bueno OFA. Relações entre controle executivo e memória episódica verbal no comprometimento cognitivo leve e na demência tipo Alzheimer. Estud Psicol. 2005; 10(1):63-71.

13. Capovilla AGS, Assef ECS, Cozza HFP. Avaliação neuropsicológica das funções executivas e relação com desatenção e hiperatividade. Aval Psicol. 2007; 6(1):51-60.

14. Saboya $E$, Saraiva D, Palmini A, Lima $P$, Coutinho G. Disfunção executiva como uma medida de funcionalidade em adultos com TDAH. J Bras Psiquiatr. 2007; 56(Supl1):30-3.

15. Butman J, Allegri RF, Harris P, Drake M. Fluencia verbal en Español. Datos normativos en Argentina. Medicina. 2000; 60(5/1):561-4.

16. Baron ID. Neuropsychological evaluation of the child. New York: Oxford University Press; 2004. p.171-85.

17. Szatkowska I, Grabowska A, Szymanska O. Phonological and semantic fluencies are mediated by different regions of the prefrontal cortex. Acta Neurobiol Exp. 2000; 60(4):503-8.

18. Tsakanikos E, Claridge G. More words, less words: verbal fluency as a function of 'positive' and 'negative' schizotypy. Person Indiv Differ. 2005; 39(4):705-13.

19. Brucki SMD, Rocha MSG. Category fluency test: effects of age, gender and education on total scores, clustering and switching in Brazilian Portuguesespeaking subjects. Braz J Med Biol Res. 2004; 37(12):1771-7.

20. Schaufelberger M, Senhorini MCT, Barreiros MA, Amaro Júnior E, Menezes PR, Scazufca M, et al. Frontal and anterior cingulate activation during overt verbal fluency in patients with first episode psychosis. Rev Bras Psiquiatr. 2005; 27(3):228-32.

21. Capovilla AGS, Capovilla FC, Suiter I. Processamento cognitivo em crianças com e sem dificuldades de leitura. Psicol Est. 2004; 9(3):449-58. 22. Knapp P, Rohde LA, Lyszkowski L, Johannpeter J. Terapia cognitivo-comportamental no transtorno de déficit de atenção/hiperatividade manual do paciente. Porto Alegre: Artmed; 2002.

23. Oosterlaan J, Scheres A, Sergeant JA. Which executive functioning deficits are associated with AD/HD, ODD/CD and comorbid AD/HD+ODD/CD? J Abnormal Child Psychol. 2005; 33(1):69-85.
24. Biederman J, Petty C, Fried R, Fontanella J, Doyle $A E$, Seidman LJ, et al. Impact of psychometrically defined deficits of executive functioning in adults with attention deficit hyperactivity disorder. Am J Psychiatr. 2006; 163(10):1730-8.

25. Cohen MJ, Morgan AM, Vaughn M, Riccio CA, Hall J. Verbal fluency in children: developmental issues and differential validity in distinguishing children with attention-deficit hyperactivity disorder and two subtypes of dyslexia. Arch Clin Neuropsychol. 1999; 14(5):433-43.

26. Chan RCK, Chen EYH. Development of a Chinese verbal fluency test for the Hong Kong psychiatric setting. J Psychiatr. 2004; 14(2):8-11.

27. Henry JD, Phillips LH. Covariates of production and perseveration on tests of phonemic, semantic and alternating fluency in normal aging. Neuropsychol Dev Cog B Aging Neuropsychol Cogn. 2006; 13(3-4):529-51.

28. Mansur LL, Carthery MT, Caramelli P, Nitrini R. Linguagem e cognição na doença de Alzheimer. Psicol Reflex Crit. 2005; 18(3):300-7.

29. Abrahams S, Goldstein LH, Simmons A, Brammer M, Williams SCR, Giampietro V, et al. Word retrieval in amyotrophic lateral sclerosis: a functional magnetic resonance imaging study. Brain. 2004; 127(7):1507-17.

30. Schwartz S, Baldo J, Graves RE, Brugger $P$. Pervasive influence of semantics in letter and category fluency: a multidimensional approach. Brain Lang. 2003; 87(3):400-11.

31. Koren R, Kofman O, Berger A. Analysis of word clustering in verbal fluency of school-aged children. Arch Clin Neuropsychol. 2005; 20(8):1087-104.

32. Villodre R, Sanchez-Alfonso A, Brines L, Nuñez $A B$, Chirivella J, Ferri J, et al. Fluencia verbal: estudio normativo piloto según estrategias de "agrupación" y "saltos" de palabras en problación española de 20 a 49 anõs. Neurol. 2006; 21(3):124-30.

33. Ardila A, Rosselli M, Matute E, Guaiardo S. The influence of the parents' educational level on the development of executive functions. Develop Neuropsychol. 2005; 28(1):539-60.

34. Ramírez M, Ostrosky-Solís F, Fernández A, Ardila-Ardila A. Fluidez verbal semántica en hispanohablantes: un análisis comparativo. Rev Neurol. 2005; 41(8):463-8.

35. Ostrosky-Solis F, Gutierrez AL, Flores MR, Ardila A. Same or different? Semantic verbal fluency across Spanish-speakers from different countries. Arch Clin Neuropsychol. 2007; 22(3):367-77.

36. Brucki SMD, Malheiros SMF, Okamoto IH, Bertolucci PHF. Dados normativos para o teste de fluência verbal categoria animais em nosso meio. Arq Neuropsiquiatr. 1997; 55(1):56-61. 
37. Hurks PPM, Hendriksen JGM, Vles JSH, Kalff AC, Feron FJM, Kroes $M$, et al. Semantic category fluency versus initial letter fluency over 60 seconds as a measure of automatic and controlled processing in healthy school-aged children. J Clin Exp Neuropsychol. Neuropsychol Develop. 2006; 28(5):684-95.

38. Coutinho G, Mattos P, Araújo C. Desempenho neuropsicológico de tipos de transtorno do déficit de atenção e hiperatividade (TDAH) em tarefas de atenção visual. J Bras Psiquiatr. 2007; 56(1):13-6. 39. Diamond A. Attention-deficit disorder (attentiondeficit/hyperactivity disorder without hyperactivity): a neurobiologically and behaviorally distinct disorder from attention-deficit/hyperactivity disorder (with hyperactivity). Dev Psychopathol. 2005; 17(3): 807-25.

40. Melo LM, Barbosa ER, Caramelli P. Declínio cognitivo e demência associados à doença de Parkinson: características clínicas e tratamento. Rev Psiquiatr Clín. 2007; 34(4):176-83.

41. Abwender DA, Swan JG, Bowerman JT, Connolly SW. Qualitative analysis of verbal fluency output: review and comparison of several scoring methods. Assessment. 2001; 8(3):323-38.

RECEBIDO EM: 04/07/2008

ACEITO EM: 16/03/2009

Endereço para correspondência:

Danielle Câmara Silveira

Rua Conde de Bonfim, 54/312

Rio de Janeiro - RJ

CEP: 20520-100

E-mail: daniellecamara@ hotmail.com 